\title{
Los beneficios de la insulina en Diabetes tipo II son mayores en pacientes con altos niveles de hemoglobina glicosilada
}

Starting insulin therapy in patients with type II diabetes. Effectiveness, complications and resource utilization. Hayward R., Manning W., Kaplan S., et al. Jama 1997; 278:1663-9.

\section{Objetivo}

Analizar la efectividad, complicaciones y utilización de recursos asociada al tratamiento con insulina en diabéticos adultos (DBT II).

\section{Diseño}

Estudio prospectivo de cohortes y estudio de corte transversal anidado.

\section{Lugar}

Plan de salud gerenciado, Seattle, EEUU.

\section{Pacientes}

Participaron 8668 pacientes. Los criterios de selección incluyeron: individuos > 30 años sin historia de cetoacidosis que usaran sulfonilureas o insulina determinados por base de datos de farmacia 0

uno de los siguientes: 1) Glucemia en ayunas $>140 \mathrm{mg} \%$ 2) una muestra aislada $>200 \mathrm{mg} \%$ o 3) hemoglobina glicosilada (HbA1c)

3 desvíos standard de la media.

\section{Evaluación de factores pronósticos}

Los datos se obtuvieron a través de encuestas, información clínica y administrativa. Además se recolectaron datos clínicos detallados de una muestra de 1738 pacientes para utilizar un sistema validado de ajuste por severidad y comorbilidad.

\section{Medición de resultados principales}

Utilización de recursos (internaciones, consultas ambulatorias, laboratorio y automonitoreo de glucemia) y control de DBT II por HbA1c.

\section{Resultados principales}

\begin{tabular}{llc}
\multicolumn{3}{l}{ Variación de indicadores según HbA1c basal } \\
\hline Valor de HbA1c basal 12 & Diferencia ajustada al año \\
meses previos a la insulina & HbA1c & Consultas \\
\hline $13 \%$ & $-1.6 \%$ & +4.8 \\
\hline $11 \%$ & $-1.1 \%$ & +3.9 \\
\hline $9 \%$ & $-0.5 \%$ & +3.0 \\
\hline
\end{tabular}

Diferencia ajustada [ IC $95 \%$ ]

\begin{tabular}{llll} 
Indicador & $\begin{array}{l}\text { Sin } \\
\text { Insulina }\end{array}$ & $\begin{array}{l}\text { Luego de un } \\
\text { Año con insulina }\end{array}$ & $\begin{array}{l}\text { Diferencia ajustada } \\
\text { [ IC 95\% ] }\end{array}$ \\
\hline Consultas/año & 9.7 & 12.6 & $+3.1[1.9$ a 4.0$]$ \\
Costo de lab/año(\$) & 86 & 107 & $+10[0$ a 21] \\
\hline HbA1C & $9.3 \%$ & $8.4 \%$ & $-1.0[-0.8$ a -1.2$]$
\end{tabular}

El inicio de insulinoterapia fue mayor cuanto más alta era la HbA1c basal. No hubo diferencias en las hospitalizaciones entre los que recibían sulfonilureas o insulina (corte transversal) o en los que iniciaron insulina durante el seguimiento.

\section{Conclusiones}

El inicio de insulina fue seguro y efectivo para lograr un control moderado en pacientes con DBT II pobremente controlada (HbA1c $>10 \%)$ pero no logró mejor control en aquellos con HbA1c entre 8 y $10 \%$ medicados con sulfonilureas. La terapia con insulina fue acompañada de un previsible y significativo aumento en la utilización de recursos.

Fuente de financiamiento: Agency for Health Care Policy and Research.

\section{COMENTARIO}

El estudio de control y complicaciones de la DBT I (1) confirmó que el control estricto de la glucemia con insulinoterapia intensiva reduce sustancialmente las complicaciones microvasculares. A partir de su publicación se ha generado una interesante controversia sobre el tratamiento de la DBT II con insulina con la idea de obtener similares beneficios. La fisiopatología de la hiperglucemia en la DBT II es diferente a la DBT I, y la insulino resistencia característica de la DBT II puede dificultar el logro de un control estricto, aún con regímenes intensivos, sin lograr ventajas comparativas con el tratamiento oral (2). Otros estudios han demostrado que el tratamiento intensivo con insulina fue factible $(3,4)$ y que redujo significativamente las complicaciones (5). En el presente trabajo se destaca el hecho de analizar la atención de diabéticos II en su situación habitual y esto permite estimar no sólo el impacto clínico de las distintas alternativas sino también los eventuales costos para el sistema de salud.

A mayor nivel basal de HbA1c, mayor fue la probabilidad de iniciar insulina en aquellos tratados con hipoglucemiantes. Sin embargo, un $60 \%$ de estos pacientes tenían $\mathrm{HbA} 1 \mathrm{C}>8 \%$ al año y un 35\% > 9\% al igual que aquellos que seguían recibiendo sulfonilureas. La respuesta a la terapia con insulina varió en función del control basal de la DBT II,

\section{Dr Fernando Rubinstein}

Unidad de Medicina Familiar y Preventiva Hospital Italiano de Buenos Aires. observándose un descenso 3 veces mayor de la HbA1c en aquellos con peor control ( $\downarrow 1.6 \%$ contra $\downarrow 0.5 \%$ en aquellos con control moderado). Si bien existe correlación entre el nivel de HbA1c y la enfermedad microvascular en la DBT I y II, la incidencia de complicaciones terminales es mucho menos frecuente en esta última. Además, el eventual papel que la hiperinsulinemia pudiera jugar en el desarrollo de la enfermedad ateroesclerótica agrega aún más datos a la controversia. Un interesante análisis de decisión (6) estimó los beneficios potenciales del tratamiento con insulina en DBT II en función de la edad de comienzo y el nivel de HbA1c y concluyó que sólo los pacientes < 50 años se beneficiarían con un control estricto de su glucemia mientras que en aquellos con comienzo más tardío el control moderado prevendría la mayoría de las complicaciones microvasculares. La recomendación de los expertos es tratar de mantener la glucemia dentro de límites casi normales e intensificar el tratamiento si la HbA1c , $8 \%$. Sin embargo la relación costo-efectividad en los > 50 años es menor dado el escaso beneficio clínico estimado (7). Debemos destacar que el tratamiento combinado de insulina e hipoglucemiantes es una alternativa que minimiza los riesgos de la hiperinsulinemia y obtiene un control deseable en pacientes con DBT II no controlados (8).

\footnotetext{
Referencias

1-The DCCT Research Group. The effect of intensive treatment of diabetes on the development and progression of Long term complications in insulin dependent diabetes mellitus. N Enc J Med.1993:329:977-98 2- Turner R. Cull C. Holman R. United Kingdom Prospective diabetes study 17: A 9 year update of a randomized controlled trial on the effect of improved metabolic control on complications in non insulin dependent diabetes mellitus.

3- Colwell JA. The feasibility of intensive insulin management in non insulin dependent diabetes mellitus. Ann Intern Med.1996:124:131-5

4- Abraira C, Colwell JA, Nuttall FQ et al. Veterans affair cooperative study on glycemic control and complications in type II diabetes. Diabetes Care. 1995 18(8): 1113-23

5- 0hkubo Y Kishikawa $\mathrm{H}$, Araki E et al. Intensive insulin therapy prevents the progression of microvascular complications in Japanese patients with non insulin dependent diabetes mellitus:A randomized prospective 6-years study. Diabetes Res. Clin. Pract.1995.28:103-17

6-Vijan S, Hofer T, Hayward R Estimated benefits of glycemic control in microvascular complications in type II diabetes. Ann Intern Med.1997;127:788-795

7- Colwell J. Controlling type II diabetes. Are the benefits worth the costs? Editorial JAMA.1997;278:1700

8- Henry R. Glucose control and insulin resistance in non insulin dependent diabetes mellitus. Ann of Intern Med.1996;124:97-103.
} 